\title{
Mixed Robotic Interface $\Gamma$ : Searching for a hybrid cyber-physical design/experience interface using virtual/actual robots
}

\author{
Ebrahim Poustinchi ${ }^{1, *}$ \\ ${ }^{1}$ College of Architecture and Environmental Design, Kent State University, Kent, Ohio, USA
}

\begin{abstract}
Mixed Robotic Interface is a project-based design-research investigation, studying new ways of creating hybridized cyber-physical design and experience interfaces, at the intersection of robotics - as its core component, and augmented reality, game design, projection mapping, and digital fabrication. Mixed Robotic Interface $\Gamma$ - as part of Mixed Robotic Interface series of research projects, focuses on using "actual" and "virtual" robot arms as a possible creative medium and extensions of design/gaming environment creating immersive atmospheres for "experiencing" design. This research questions the possibilities of creating an architectural/spatial atmosphere through digitally enhanced experiences. Different from some of the current experiments with augmented reality (AR), virtual reality (VR) and projection-mapping in architecture, Mixed Robotic Interface $\Gamma$ is not looking into "immersive" experience as a way to "blur" the boundaries of digital and physical - similar to virtual reality experience with headsets. Instead, Mixed Robotic Interface $\Gamma$ creates a recognizable gap between real and virtual to open up a creative space for the user/audience to be involved between these two mediums. Mixed Robotic Interface $\Gamma$ uses para-fictional storytelling as a way to engage the audience with the experience and to create continues atmospheric qualities.
\end{abstract}

\section{Introduction}

Almost immediately after the introduction of digital tools for design in the early 1990s, the question of bridging this medium to the physical word has been one of the central demands in the field of digital design. Introduction of computer numerical control (CNC) machines, followed by the presence of robotic arms as part of the fabrication facility in design set-ups, made this communication between the digital environment and physical world happened. This bridging brought up the question of the role of technology — specifically robot arms, not only as a fabricator (as they have been intended to be used in the past couple of decades), but as possible physical extensions for digital design/experience platforms.

\footnotetext{
*Corresponding author: mpoustin@kent.edu
} 
On the other hand, the rapid development of immersive user experience (UX) platforms such as XR (Augmented, Virtual and Mixed reality) and interactive projection mapping, made it possible to "project"/overlay the digital content directly onto the physical world to create hybrid curated atmosphere.

Mixed Robotic Interface $\Gamma$ research aims to study user experience through storytelling and environmental ambiance, and uses both a robotic arm (both virtual and actual) and immersive experience platforms - specifically AR and projection mapping. The "Impossible Objects" design series by Kruysman-Proto and Curime Batliner [1] and the "Aether Project" by Guvenc Ozel studio at University of California Los Angeles [2] are some examples of using a combination of projection mapping and robot arms to create "qualities" and ambiance. Another familiar example of the use of robotics in conjunction with projection mapping to curate an atmospheric spatial effect is the work of Bot and Dolly-creative robotic studio in designing and shooting special effects of the "BOX" [3].

\section{Mixed Robotic Interface $\Gamma$}

Mixed Robotic Interface $\Gamma$ follows Sylvia Lavin's "Man vs. Machine" retooling creativity exhibition conversation, where Lavin asserts that increasingly larger amounts of creative resources are being put into producing new tools and concepts that are designed not to make things but, to amplify the creative capacities of others [4]. Similarly, in his article "Postarchitecture," Guvenc Ozel calls for a combination of virtual and actual, and argues that our surrounding "space" is a hybrid of both physical materials and electronic bits [5].

Mixed Robotic Interface $\Gamma$, as a design research investigation, looks into new technologies and their possibility to become mediums for experiencing atmospheric effects of design.

Enhanced with both augmented reality techniques and projection mapping methods, through video/animation making, storytelling, cellphone-application developments, digital and experimental fabrications, and "super-graphics," Mixed Robotic Interface $\Gamma$ presents two projects: "Robotic Pool Party!" and "Child Robot". Both projects look closely at the issue of cutting-edge technology in conjunction with storytelling and "user" experience and focus on three central themes:

- Storytelling through para-fictional scenarios, where a technological icon-an industrial robot in this research, becomes one of the core storytellers.

- Creating curated ambiance/experiences through seamless (or purposely "seemed") cyber-physical interfaces.

- Revisiting the potential of projection-mapping and augmented reality - as existing techniques for immersive design, to create custom atmospheres considering current contemporary discourse in architecture through classical issues/conditions such as poche, corner, envelope and new concepts of part-to-part and part-to-whole relationship, considering flat architectural ontology as suggested by Tom Wiscombe [6].

\subsection{Mixed Robotic Interface Г project I: ROBOTIC POOL PARTY!}

Designed and exhibited as part of the 3-Ways show curated by Anthony Morey, Ivan Bernal and Ryan Tyler Martinez, at the A+D Architecture and Design Museum in Los Angeles, the "Robotic Pool Party!" project revisits the potential of virtual animated robots and projection 
mapping as a method for experiencing the space and creating atmosphere through story telling (Figure 1).

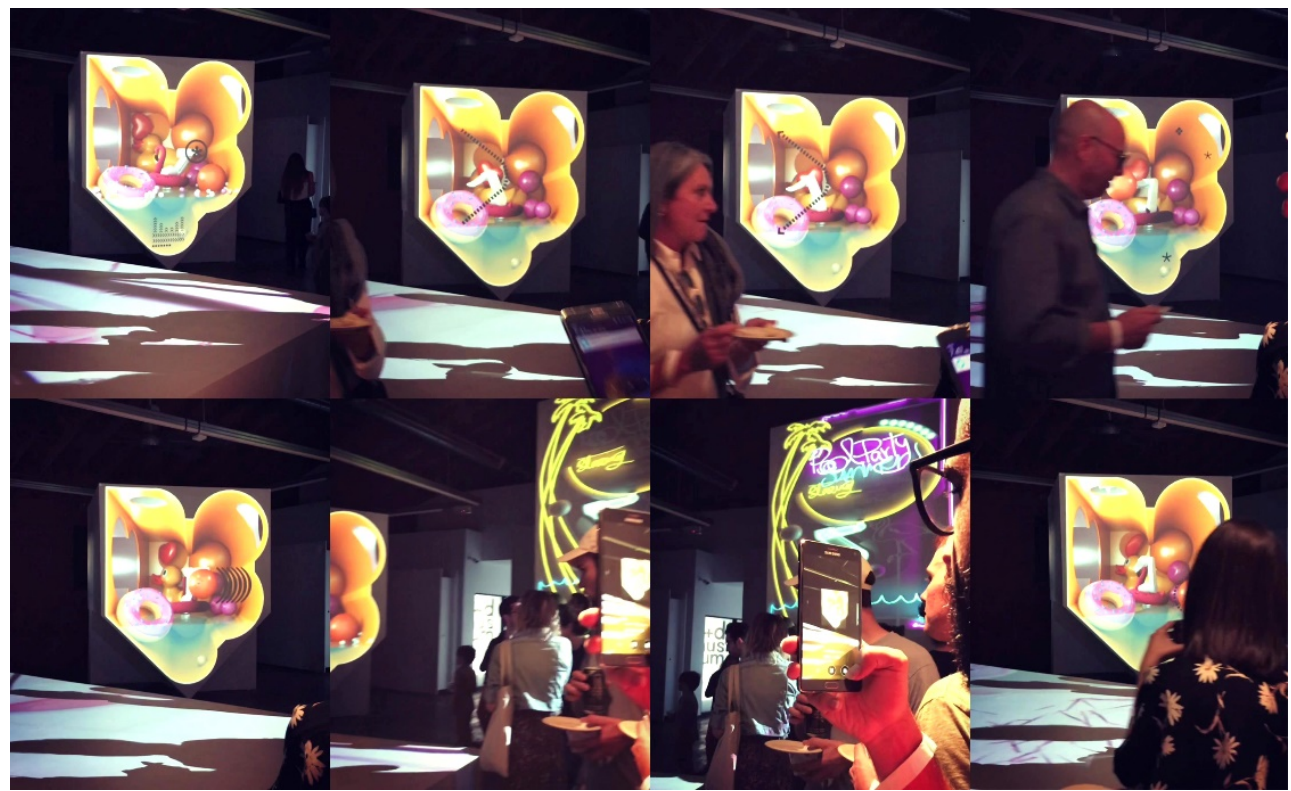

Fig. 1. Photos from the installation set-up at the $A+D$ Architecture and Design Museum in Los

Angeles. The animation is projected on a physical "canvas" provided by the museum and has become a window to a new ambiance.

Using animation as a story-telling medium, "Robotic Pool Party!" uses a combination of realistic and fake representational techniques to achieve a unique mixed atmosphere. Realistic physic simulations of Autodesk MAYA in conjunction with Inverse Kinematic (IK) techniques - to animate the robot on one hand, and highly saturated plastic/toy like qualities of the materials - even water, on the other hand, creates a blended yet divergent mixture of qualities to craft a unique story that lives between mediums (Figure 2).

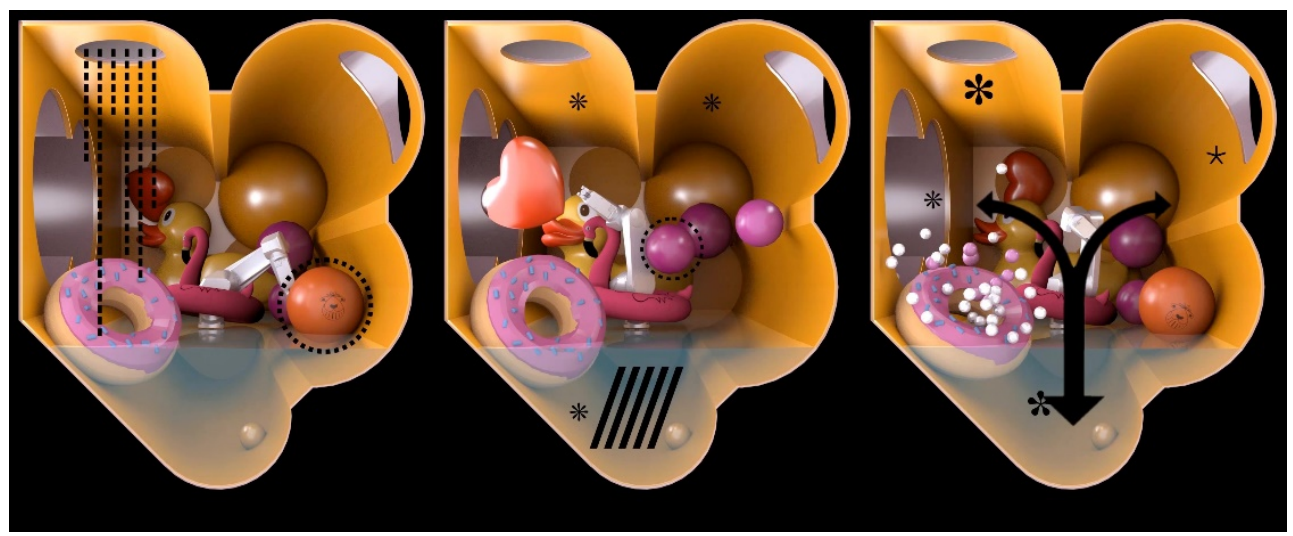

Fig. 2. Frames from the animation, illustrating the highly saturated plastic/toy like qualities of the materials in the scene.

Migrated from a factory assembly line, the robot at this pool party becomes a "humanoid," and the ultimate character of the "story". Celebrating the friction between actual and virtual, and real and projected, and interacting with its surroundings, the robot—in combination with its context, becomes a medium in which, the audiences, experience the atmosphere of the 
installation. The robot plays (interacts) with inflatables in the pool augmented with humanistic gestures and characteristics, as a way to engage the audience into the "performance" and story of the installation. In addition and through poche and sectional studies, "Robotic Pool Party!" also examines the potential of fake-depths created using projection mapping and in contrast with the project's three-dimensional canvas. Using nonrealistic representational techniques, audience can clearly distinguish the projected/fake depth. However, this detachment is being used as part of the story to ultimately increase the engagement of the audience throughout the installation.

To architectural respond to the idea of celebrated representational detachment between the projected content (virtual) and the physical context of the show (actual), the project uses the notion of object-inside-an-object as Tom Wiscombe describes. "Robotic Pool Party!" aims to amplify the atmospheric contrast between the dark plain cubic physical canvas of the projection and the vivid, playful, dynamic and active projection content. The Section is dynamically changing to illustrate the difference between the outside and "inside" of the created depth as a way to engage the audience in the story/atmosphere that the project is aiming to develop (Figure 3).
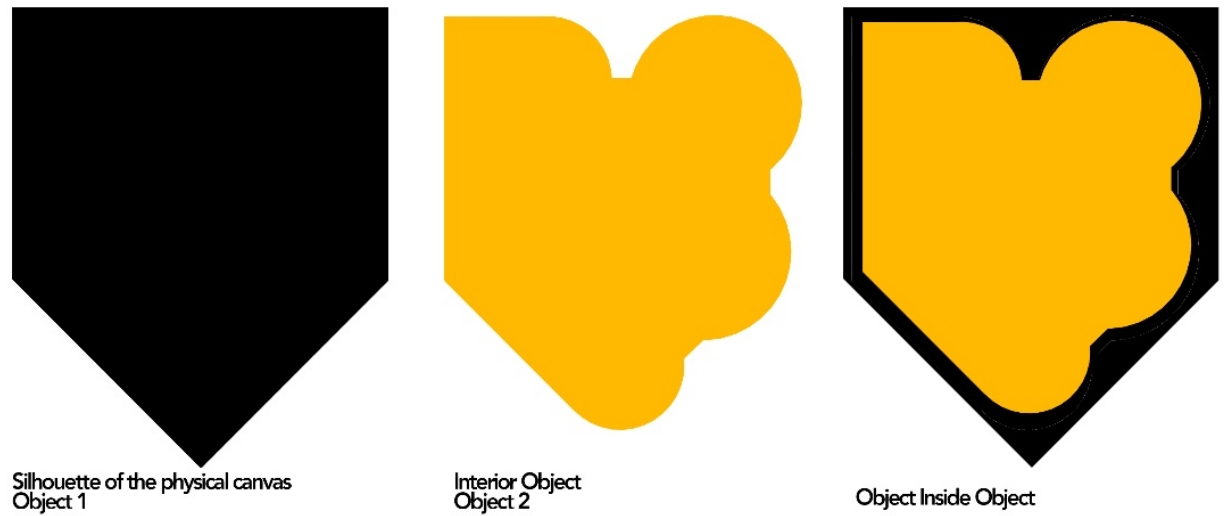

Fig. 3. The use of poche as a way to amplify the atmospheric contrast between the outside and inside.

\subsection{Mixed Robotic Interface Г project I: Child-Robot!}

The Child-Robot project studies the relationship between actual and virtual-through storytelling as a bridge, and using a robotically animated hybrid-digital-physical scene/stage as the vehicle. Using multiple techniques and platforms ranging from custom augmented reality cell-phone applications, custom robotically camera controlling platform, green-screening techniques and super-graphics as spatial connectors, the Child-Robot project redefines the use of augmented reality techniques, not as a seamless "immersion" tool, but as a gateway to a story that is happing in two different worlds: the world of the physical robot and the world of the digital animation overlaid through AR (Figure 4). 


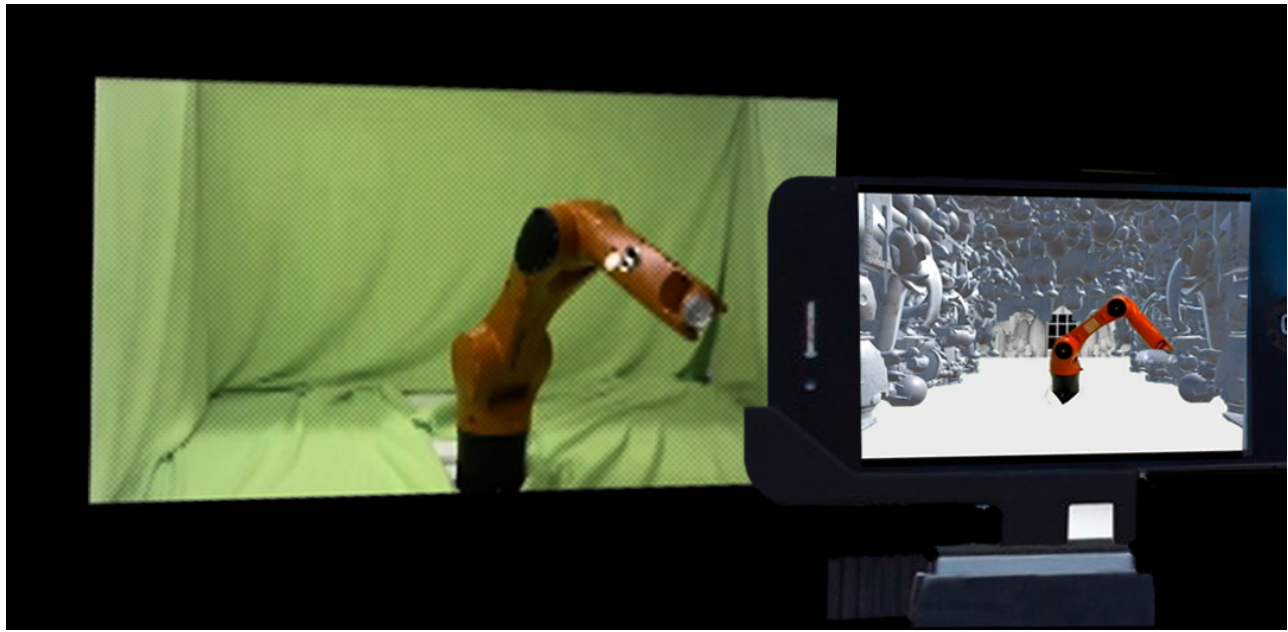

Fig. 4. The cell phone AR application creates a mixture of realities with combining the virtual room and the actual footage of the robot arm.

The story is set in a para-fictional scenario on the speculation of a future child's room where the robot arm plays the role of the child to illustrate a futuristic atmosphere and as way to initiate the story. The set-up is the result of a precise calibration of the physical robot arm, the virtual room and the virtual animated kit-bash scene (child-robot room) to enhance the experience. Using augmented reality techniques, Child-Robot enables the users to experience a mixture of reality, fantasy, actual and virtual through a custom-made software application and an actual scene. The audience can move around the robot in action, and through the lens of the device, overlay the customized context of the story to enrich the experience/performance of the robot as the actor (Figure 5).

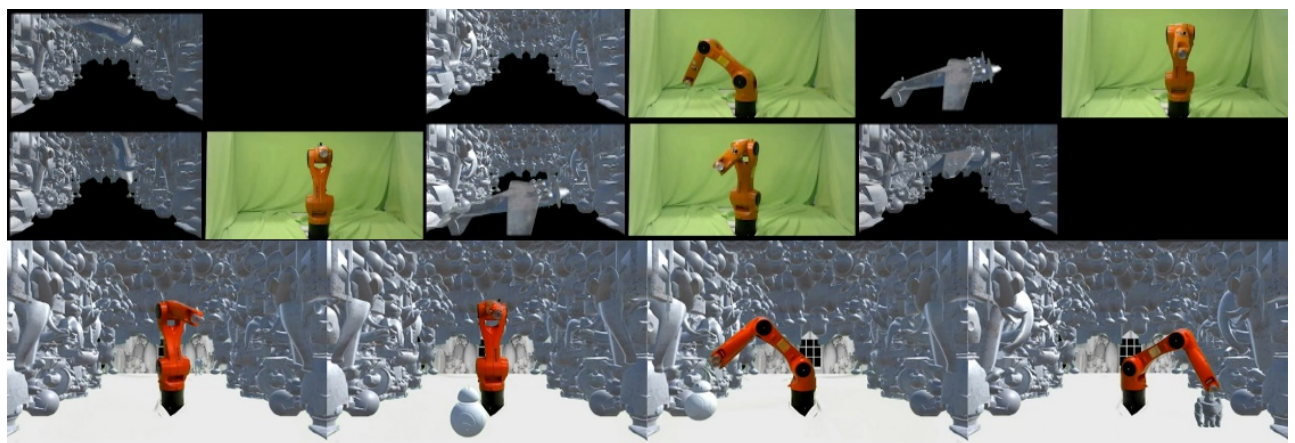

Fig. 5. Screen captures from the robotic scene experience through regular and augmented cameras. The robot becomes the performer of the story and the AR environment, amplifies the atmospheric effects of the story/performance by introducing a toy-based.

The audience can move back and forth between the actual robot/physical set-up and the virtual/augmented environment, with which the robot is interacting. Using Oriole-custommade robotic videography and controlling plug-in for Grasshopper 3D, Child-Robot also examine the potential of a hybrid digital-physical set-up to create, curate, tell and visualize a story from both robots' perspective and the audience/users (Figure 6). 

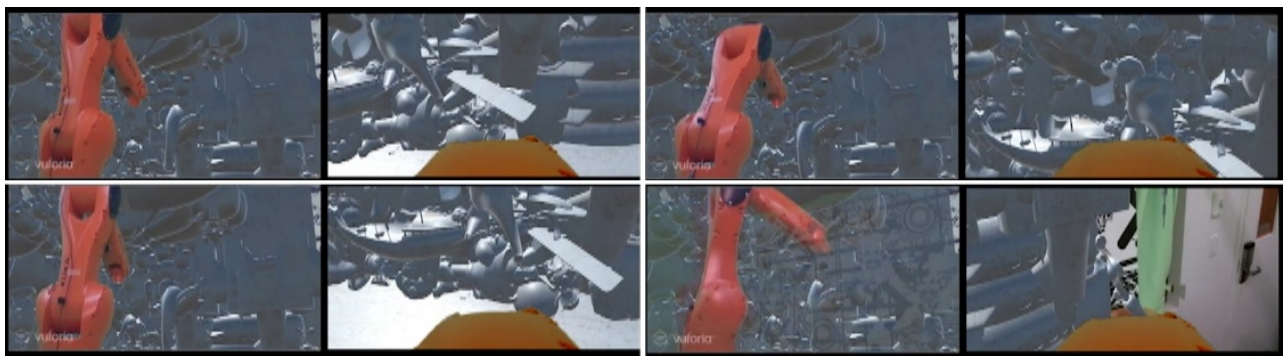

Fig. 6. Screen captures from the robot perspective. Using Oriole plug-in for the grasshopper, the audience was able to also see through the eyes of the robot, as the leading "actor" in the science.

The Child-Robot project questions the potential of hybrid digital-physical platforms as not only design, representation, and simulation tools but ultimately as a source for spatial experience and storytelling where digital and physical, augmented and real, and artificial and natural blend into a distinguishable mixture.

\section{Conclusion}

The Mixed Robotic Interface $\Gamma$ examined the potential of storytelling as a medium for immersion. Using robots as "programmable" actors that "think" digitally and "act" physically, and through AR and projection mapping techniques as transitional "windows" between actual and virtual, we observed that initial studies-Robotic Pool Party and Child Robot projects created engaging experiences for the audience through a hybrid experience medium. Going back and forth between multiple representational and atmospheric set-ups, and having the ability to immerse into the digital experience without losing the connection to the physical environment, we observed through informal conversations and questioners that our audience fund Mixed Robotic Interface $\Gamma$ different from conventional AR or projection mapping. The main difference was that in this research projects, instead of the tool/device - virtual reality or mixed reality headsets, the story became the primary tool to blur the boundaries between actual and virtual.

\section{Limitation | Delimitations, Future Research Plans}

Mixed Robotic Interface research in general and Mixed Robotic Interface $\Gamma$ in particular are very young research intonations that are seeking new mediums for design, communication, experience, and storytelling. One of the primary limitations of the current research is the limited number of users and limited feedback from them. Currently, we are looking into more proper data-collection techniques - such as human factor measurement or questioners, as a way to precisely compare the differences between Mixed Robotic Interface $\Gamma$ and other existing methods. Another parameter of this research that we would like to develop further is the use of Mixed Robotic Interface method as an architectural design tool to generate from, space and compositions based on the interaction of the audience with the "mixed" story.

Currently, we are working on expanding the research, looking into set-ups with multiple robots where both the physical space and the digital "projection" are equally dynamic and performative. 


\section{References}

1. B. Kruysman and J. Proto, "Impossible objects," Architectural Design, no 227, ed. Bob Sheil, 106-111, (January/February 2014).

2. G. Ozel, "Case for an Architectural Singularity: Synchronization of Robotically Actuated Motion, Sense-Based Interaction and Computational Interface," in Proceedings of the 34th Annual Conference of the Association for Computer Aided Design in Architecture (ACADIA), ed. David Gerber, Alvin Huang, and Jose Sanchez, 399-408, (2015)

3. Bot \& Dolly Fuses 3D Animation and Industrial Automation, (2013, November 25),

4. Retrieved from https://www.roboticsbusinessreview.com/consumer/bot_dolly_fuses_3d_animation_an d industrial_automation/

5. S. Lavin, (2015, July 13). "Man vs. Machine: Sylvia Lavin Retools Creativity." Los Angeles Forum for Architecture and Urban Design. Retrieved from http://laforum.org/delirious/man-vs-machine-sylvia-lavin-retools-creativity/

6. G. Ozel, “Toward a postarchitecture," Log 36, 99-105, (Winter 2016)

7. T. Wiscombe, Discreteness or towards a flat ontology of architecture. Project, (Issue 3), 34-43, (2014). 